\title{
Revisión del estado del arte para la gestión y mejora de los procesos empresariales
}

\section{(Revision of the state of the art for the administration and improvement of the managerial processes)}

\author{
Henrry Ricardo Cabrera ${ }^{1}$, Alberto Medina León², Dianelys Nogueira Medina², Quirenia \\ Núñez Chaviano ${ }^{1}$
}

\begin{abstract}
Resumen:
El presente artículo relaciona conceptos y conocimientos sobre la tendencia internacional en cuanto a la gestión y mejora de los procesos empresariales. En la metodología se consideró la revisión y análisis de publicaciones de acceso online, bases de datos indexadas y otras de la Web de la Ciencia para identificar aquellos aspectos de interés que influyen en el desempeño de las empresas y sus procesos. Los principales hallazgos indican que en la actualidad cualquier intento por formalizar la gestión y mejora de procesos debe contener supuestos teóricos alrededor de la gestión por procesos y la integración de sistemas, además es imprescindible aceptar como base las buenas prácticas de gestión, tratadas con un enfoque de procesos, que resaltan en la selección de los procesos, la determinación y evaluación de las oportunidades de mejora, los indicadores y su relación con las estrategias empresariales.
\end{abstract}

Palabras clave: Mejora de procesos; Sistemas de gestión empresariales; Integración de sistemas de gestión.

\begin{abstract}
:
The present article relates concepts and knowledge on the international tendency as for the administration and improvement of the managerial processes. In the methodology review and analysis published online access, indexed database and other Web of Science is considered to identify those aspects of interest that influence the performance of companies and their processes. The main findings indicate that today any attempt to formalize the management and process improvement must contain theoretical assumptions about process management and system integration, it is also imperative to accept the basis of good management practices, treated with a focus process, highlighted in the selection process, the identification and assessment of opportunities for improvement, indicators and its relationship with business strategies.
\end{abstract}

Keywords: Process improvement; Business management systems; Integration of management systems.

\footnotetext{
${ }^{1}$ Universidad de Cienfuegos, Cienfuegos - Cuba( \{hricardo, knunez\} @ucf.edu.cu)

2 Universidad de Matanzas, Matanzas - Cuba ( \{alberto.medina, dianelys.nogueira\} @umcc.cu )
} 


\section{Introducción}

Desde finales del siglo veinte hasta la fecha, el mundo ha sufrido numerosos cambios que van desde la consolidación de la globalización de los mercados hasta la revolución en la tecnología de la información y las comunicaciones(Alonso Torres, 2014).

(2.1) Por tanto, la gestión de las empresas, en la creciente complejidad de sus actividades, debe procurar la preparación de los componentes humanos, y la mejoría de los materiales. Resultan comunes los logros obtenidos en la gestión sobre la base de un enfoque por proceso(Nariño Hernández, Nogueira Rivera, \& Medina León, 2014), fundamentalmente en el desarrollo de una cultura orientada a la mejora continua, la sistematización de los procesos, la participación del personal, el trabajo en equipo y la creatividad(Hernández Nariño, Medina León, Nogueira Rivera, \& Marqués León, 2009).

(2.2-2.3) La calidad de productos y/o servicios constituye un elemento importante en la supervivencia (Karapetrović, Casadesus, \& Heras, 2010)(Gasiorowski, 2013) y posicionamiento de las empresas en el mercado (Bratić, 2011). El estudio de la calidad ha evolucionado, de un inicio, centrado en el control de la calidad a, finalmente, la implementación de la Calidad Total y a sistemas de gestión empresariales estrechamente relacionados con la mejora continua.

(2.4) Las metodologías y herramientas de mejora impactan sobre las personas e introducen modificaciones en sus actitudes, aptitudes, comportamientos(Karapetrovic, 2003) (Abab Puente, Vilajosana Crusells, \& Dalmau, 2014) y conllevan a un mejor aprovechamiento de los recursos de uno u otro tipo. Se proponen y diseñan para incrementar los resultados de los indicadores de gestión(IFA, 2010)(Cuendias de Armas et al., 2013) de eficiencia, de eficacia y mejorar los resultados para todos los grupos de interés de la empresa; así repercuten sobre los resultados claves de la organización(Medina León, Nogueira Rivera, \& Hernández Nariño, 2012).

Existen numerosos procedimientos y herramientas que permiten gestionar y obtener resultados en la mejora de procesos empresariales(Ricardo Cabrera, 2010), aplicables según las características de cada empresa. Estos para su desarrollo se apoyan en el levantamiento de las oportunidades de mejoras, necesitan de un equipo de trabajo que refuerce el liderazgo(Medina León, Nogueira Rivera, Hernández Nariño, \& Viteri Moya, 2010)(Hernández Nariño, Nogueira Rivera, Medina León, \& Marqués León, 2013) y actúe sobre el papel a desarrollar por los líderes (Comas Rodríguez, Nogueira Rivera, Medina León, Romaro Bartutis, \& Lumpuy Rodríguez, 2014). Requieren además, de un plan formalizado para llevarlas a cabo y deben estar al servicio de los objetivos de la organización(Medina Léon et al., 2014).

(2.5-2.6) De un lado la evolución de los mercados y la necesidad de mejorar cada día, del otro, la importancia de satisfacer las distintas partes interesadas que influyen en el desenvolvimiento de las actividades empresariales, obligan a pensar en gestionar y mejorar los procesos en 
contribución a la integración de los diferentes sistemas normalizados. Cada día son más las empresas que integran los sistemas en un único sistema de gestión (Abab Puente et al., 2014) pues reconocen sus ventajas.

De aquí que el presente artículo consista en una revisión bibliográfica, que sobre la base de los antecedentes descritos, tiene como propósito indagar en los conceptos y saberes que relacionan la gestión y mejora de los procesos empresariales en contribución a la integración de los sistemas normalizados. Por tanto, su principal objetivo sea dotar al lector de los conocimientos básicos para comenzar a gestionar y mejorar los procesos empresariales bajo esta concepción.

\section{Desarrollo}

\subsection{Los procesos empresariales, su gestión}

Frente a la turbulencia y a la dinámica del mercado actual, muchas organizaciones responden con nuevos productos y no con el mejoramiento de sus procesos. Con el tiempo la ausencia de atención a los procesos trae consigo obsolescencia, altos costos y falta de adaptación a las demandas, acotaba Juran a comienzos de siglo (Juran \& Blanton, 2001)

También Trischler mencionaba que: el éxito de toda organización depende cada vez más de que sus procesos empresariales estén alineados con su estrategia, misión y objetivos. Además, los individuos de la organización deben comprender la importancia de su rol en el alcance de los objetivos empresariales(Trischler, 2000). Por lo tanto, cada día es más importante que los directivos intervengan en los procesos adecuados para ayudar a las personas a afrontar los cambios necesarios en el camino a la excelencia empresarial.

Se pueden identificar distintas filosofías o tendencias de amplia difusión en el mundo empresarial que reconocen la importancia de los procesos, entre ellas: el Modelo de la Fundación Europea para la Gestión de la Calidad (EFQM), el Cuadro de Mando Integral (CMI), la mejora continua, las Normas ISO, el Benchmarking, el uso de metodologías para la determinación de los Sistemas de Análisis de Riesgos y Puntos Críticos de Control (HACCP), la gestión por el conocimiento, la gestión por competencias y la integración de sistemas(Azcanio García, Quintana Tápanes, Medina León, \& Nogueira Rivera, 2006). A los anteriores también se le añade el enfoque de American Productivity \& Quality Center(APQC) (APQC, 2012), este, junto al proporcionado por la EFQM constituyen los modelos para la clasificación de procesos empresariales más difundidos en la literatura.

Se puede percibir la importancia que los distintos autores le otorgan a los procesos y su gestión, como ente fundamental para lograr la competitividad empresarial. Acotaba Amozarrain que: "las empresas son tan eficientes como lo son sus procesos" (Amozarrain, 1999), comprendiéndose porque la Gestión por Procesos constituye una de las tendencias empresariales modernas de mayor aceptación a nivel mundial. 
La Gestión por Procesos parte de la necesidad de alinear los procesos con la estrategia, la misión y los objetivos de la institución (Moreira, 2013)(Alonso Torres, 2014), y el fundamental protagonista en la ejecución son los propios procesos empresariales (Ricardo Cabrera, 2009)(Álvarez Suárez, 2011). Por ello, el punto de análisis lo constituye la gestión de la empresa basada en los procesos que la integran(Balcázar \& Lagunas, 2013)(Hernández Nariño et al., 2013).

De aquí que, el enfoque de procesos sea hoy una herramienta tan poderosa por su capacidad de contribuir de forma sostenida a los resultados (Cordoví Cuza, 2013), siempre que tenga presente a sus clientes al diseñar y estructurar sus procesos (Nogueira Rivera, 2002)(Nariño Hernández et al., 2014).

Para lograr la efectividad en cuanto a la gestión de los procesos, la empresa debe tener los procesos bien identificados (Hernández, 2003). Con esto además, alcanza mejorar el desempeño (eficiencia y eficacia) y posibilita diseñar, modelar, organizar, documentar y optimizar de forma continua su mejora.

\subsection{La gestión por procesos como ente esencial en el mejoramiento}

Las organizaciones a nivel internacional cambian su enfoque administrativo y de dirección funcional a uno basado en procesos y aunque no es un fin en sí mismo, es un medio para que las organizaciones puedan asumir de forma eficiente y eficaz sus objetivos (Medina León et al., 2010).

Como se muestra en la Tabla 1, la literatura encierra varios conceptos sobre lo que se entiende como gestión por procesos. Se pude apreciar como entre los años 1999 y el 2009 aparecen la mayor cantidad de conceptos publicados.

Tabla 1: Conceptos relacionados a la Gestión por Procesos. Fuente: En contribución a (Medina León et al., 2010)

\begin{tabular}{|l|l|}
\hline Autor & Concepto \\
\hline $\begin{array}{l}\text { (Davenport, } \\
1993)\end{array}$ & $\begin{array}{l}\text { Conjunto de } \\
\text { y organizadas destinadas a resultar en un producto específico para un determinado } \\
\text { cliente } \\
\text { Ordenamiento especifico de las actividades de trabajo en tiempo y espacio, con } \\
\text { comienzo y fin, entradas y salidas claramente identificadas en una estructura para la } \\
\text { acción. }\end{array}$ \\
\hline $\begin{array}{l}\text { Generalización de la gestión de un proceso y se aplica a una organización en su } \\
\text { conjunto. }\end{array}$ \\
\hline $\begin{array}{l}\text { (Amozarrain, } \\
1999)\end{array}$ & $\begin{array}{l}\text { Gestionar toda la organización basándose en los Procesos era entender estos como } \\
\text { una secuencia de actividades orientadas a generar un valor añadido sobre una } \\
\text { entrada para conseguir un resultado, y una salida que a su vez satisfaga los } \\
\text { requerimientos del Cliente }\end{array}$ \\
\hline $\begin{array}{l}\text { (Zaratiegui, } \\
1999)\end{array}$ & $\begin{array}{l}\text { El éxito de toda organización depende, cada vez más, de que sus procesos } \\
\text { empresariales estén alineados con su estrategia, misión y objetivos. Detrás del } \\
\text { cumplimiento de un objetivo, se encuentra la realización de un conjunto de actividades } \\
\text { que, a su vez, forman parte de un proceso. Es por ello que el principal punto de } \\
\text { análisis lo constituye, precisamente, la gestión de la empresa basada en los procesos } \\
\text { que la integran para diseñar y estructurar en interés de sus clientes. }\end{array}$ \\
\hline Se enmarca en la Gestión de la Calidad. Supone reordenar los flujos de trabajo. \\
\hline
\end{tabular}




\begin{tabular}{|c|c|}
\hline denas, 2000) & \\
\hline $\begin{array}{l}\text { (Junginger, } \\
2000)\end{array}$ & $\begin{array}{l}\text { la forma de reaccionar con más flexibilidad y rapidez a cambios en las condiciones } \\
\text { onómicas. }\end{array}$ \\
\hline $\begin{array}{l}\text { Colegio Oficial } \\
\text { de Ingenieros } \\
\text { Superiores } \\
\text { Industriales de } \\
\text { la Comunidad } \\
\text { Valenciana. } \\
\text { (2001) }\end{array}$ & $\begin{array}{l}\text { Concentra la atención en el resultado de cada uno de los procesos que realiza la } \\
\text { empresa, en lugar de en las tareas o actividades. }\end{array}$ \\
\hline $\begin{array}{l}\text { (Aiteco } \\
\text { Consultores, } \\
\text { 2002) }\end{array}$ & $\begin{array}{l}\text { Percibe la organización como un sistema de procesos que permiten lograr la } \\
\text { satisfacción del cliente. Fundamenta una visión alternativa a la tradicional } \\
\text { caracterizada por estructuras organizativas departamentales. }\end{array}$ \\
\hline $\begin{array}{l}\text { (Díaz } \\
\text { 2002) }\end{array}$ & $\begin{array}{l}\text { Optimiza la satisfacción del cliente, la aportación de valor y la capacidad de respuesta } \\
\text { de una organización. }\end{array}$ \\
\hline $\begin{array}{l}\text { (Mora Martínez, } \\
\text { 2002) }\end{array}$ & $\begin{array}{l}\text { Percibe la organización como un sistema interrelacionado de procesos que } \\
\text { contribuyen conjuntamente a incrementar la satisfacción del cliente. Supone una visión } \\
\text { alternativa a la tradicional caracterizada por estructuras organizativas de corte } \\
\text { jerárquico - funcional. }\end{array}$ \\
\hline $\begin{array}{l}\text { (Paneque Sosa, } \\
\text { 2002) }\end{array}$ & $\begin{array}{l}\text { Se conforma como una herramienta encaminada a conseguir los objetivos de la } \\
\text { Calidad Total, con visión centrada en el paciente (trabajo para la salud), requiere de la } \\
\text { implicación de las personas para provocar el cambio en la organización, se sustenta } \\
\text { en la búsqueda de las mejores prácticas y de un sistema de información integrado. }\end{array}$ \\
\hline $\begin{array}{l}\text { Comité } \\
\text { Institucional de } \\
\text { Acreditación. } \\
\text { Universidad de } \\
\text { Córdoba. }\end{array}$ & $\begin{array}{l}\text {-Direccionamiento hacia los objetivos de la Universidad y sus programas. } \\
\text {-Búsqueda permanente de la excelencia académica. } \\
\text {-Aumento de la eficacia y la eficiencia. } \\
\text {-Auto evaluación y evaluación externa con indicadores de alta calidad. }\end{array}$ \\
\hline $\begin{array}{l}\text { (González } \\
\text { Méndez, 2002) }\end{array}$ & $\begin{array}{l}\text { porciona una manera más realista y simple de ver y dirigir la empresa, se } \\
\text { fanizan los flujos de trabajo y las relaciones en la secuencia de clientes internos, se } \\
\text { dencia el rol de cada quién respecto al objetivo común, ayuda a clarificar para el } \\
\text { sonal y el cliente la obra entera, los pasos y la transformación de entradas en } \\
\text { das, viabilizando las percepciones y evaluaciones de roles y actuaciones y el } \\
\text { cubrimiento y solución de cualquier hecho que detenga o restrinja los flujos de } \\
\text { ión. }\end{array}$ \\
\hline $\begin{array}{l}\text { (SESCAM, } \\
2002)\end{array}$ & $\begin{array}{l}\text { ional, y en el que } \\
\text { Sobre su mejora se }\end{array}$ \\
\hline $\begin{array}{l}\text { (Ponjuán Dante, } \\
\text { 2005) }\end{array}$ & $\begin{array}{l}\text { Gestionar integralmente cada una de las transacciones o procesos que la organización } \\
\text { realiza, no sólo pensar en cómo hacer mejor lo que está haciendo (división del } \\
\text { trabajo), sino ¿Por qué? y ¿Para quién? Lo hace; puesto que la satisfacción del } \\
\text { usuario, cliente interno o externo viene determinado más por el coherente desarrollo } \\
\text { del proceso en su conjunto que el de cada función individual o actividad. }\end{array}$ \\
\hline $\begin{array}{l}\text { (ISO } \\
2008) .\end{array}$ & $\begin{array}{l}\text { ación de un sistema de procesos dentro de la organización, junto con la } \\
\text { ción e interacciones de estos procesos, así como su gestión para producir el } \\
\text { deseado, puede denominarse como "enfoque basado en procesos" }\end{array}$ \\
\hline $\begin{array}{l}\text { (Bravo } \\
\text { Carraso }\end{array}$ & $\begin{array}{l}\text { "La gestión sistémica de procesos identifica a los procesos de la empresa para } \\
\text { agregar valor a los clientes y cumplir con la estrategia del negocio" }\end{array}$ \\
\hline $\begin{array}{l}\text { (Pepper } \\
\text { Bergholz, 2011) }\end{array}$ & $\begin{array}{l}\text { e persigue la mejora continua de las } \\
\text { dentificación, selección, descripción, }\end{array}$ \\
\hline $\begin{array}{l}\text { (Maldonado, } \\
\text { 2011) }\end{array}$ & $\begin{array}{l}\text { Una gestión generadora de valor para el cliente. Determina que procesos necesitan } \\
\text { ser rediseñados o mejorados, establece prioridades y provee de un contexto para } \\
\text { iniciar y mantener planes de mejora que permitan alcanzar objetivos perseguidos. }\end{array}$ \\
\hline $\begin{array}{l}\text { (Rey } \\
2012)\end{array}$ & $\begin{array}{l}\text { Se basa en la modelación de los sistemas como un conjunto de procesos } \\
\text { interrelacionados mediante vínculos causa-efecto. Se desarrollan de forma } \\
\text { coordinada, mejorando la efectividad y la satisfacción de todas las partes interesadas. }\end{array}$ \\
\hline
\end{tabular}

Resulta interesante como Bravo Carrasco (2009) relaciona la administración del cambio, la responsabilidad social, el análisis de riesgos y un enfoque integrador entre estrategia, personas, 
procesos, estructura y tecnología, con la gestión por procesos, ubicándola como eje integrador. De aquí sugiere principios tales como: responsabilidad social, planificación estratégica, alineamiento de intereses, orientación al cliente y análisis de riesgos para el alcance de la excelencia empresarial.

Según la ISO 9001:2008, sostenido también en la nueva norma ISO 9001:2015(ISO, 2015)(Martínez Llebrez, 2015), una ventaja del enfoque basado en procesos es el control continuo que proporciona sobre los vínculos entre los procesos individuales dentro del sistema, así como su combinación e interacción.

De los conceptos recopilados en la tabla 1 se puede apreciar la importancia asignada por los distintos autores a los procesos y cómo las acciones emprendidas en ellos pueden determinar los resultados finales, incluidos los requerimientos de las distintas partes interesadas que influyen en la gestión empresarial. El aportado por Amozarrain se acepta como uno de los más completos.

La Gestión por Procesos a pesar de sus reconocidas ventajas, en varias ocasiones resulta difícil lograr su implantación; debido fundamentalmente a lo complicado que es para el hombre romper con esquemas tradicionales mantenidos por siglos o simplemente por la aparición de la resistencia natural al cambio.

Sin embargo se considera exitoso al menos, lograr la mejora con un enfoque de Gestión por Procesos, capaz de transformar la cultura empresarial hacia el reconocimiento en cada actividad, de quiénes son sus clientes, sus necesidades y exigencias; así como el logro de la adecuada coordinación, sin feudos y fincas, que permitan la concreción de los objetivos estratégicos(Medina León, et al., 2012).

También en las normas internacionales (ISO, 2009)(ISO, 2015) se fomentan la adopción de este enfoque cuando se implementa y mejora el Sistema de Gestión de Calidad, concentrándose en:

1. La comprensión y el cumplimiento de los requisitos de los clientes de cada proceso.

2. La necesidad de considerar y de planificar los procesos en términos que aporten valor (el cliente no debe pagar por algo que no le aporte valor).

3. El control, la medición y la obtención de resultados del desempeño y de la eficacia de los procesos.

4. La mejora continua de los procesos con base en mediciones objetivas.

5. Habilidad para centrar los esfuerzos en los procesos claves.

6. La integración y alineación de los procesos, que mejor alcancen los resultados deseados.

7. Aumentar la capacidad de usar los mismos recursos.

En coincidencia con (Solé Cabanes, 2012) las empresas interesadas en implantar sistemas de gestión de la calidad (añadir que también otros sistemas de gestión) o para dar paso más 
adelante, en los modelos de excelencia empresarial, deben implantar la gestión por procesos de forma efectiva y llevarlo a su documentación, metodologías, controles, actividades y recursos.

Otros autores (Medina León, $A$ et al., 2012) reconocen la utilidad que tiene la asignación de responsables de procesos y la gestión generadora de valor que este enfoque propone, para alcanzar objetivos establecidos y hacer posibles la comprensión del modo en que están configurados los procesos de negocio, de sus fortalezas y debilidades.

Por tanto, la gestión por procesos es un punto esencial en la mejora, fundamentalmente por la oportunidad que brinda de gestionar de manera eficaz los procesos empresariales. Sea en una empresa de manufactura o en los servicios, proporciona información necesaria para identificar los que necesitan ser mejorados o rediseñados.

\subsection{La mejora de los procesos. Generalidades}

Harrington declara que: "Independientemente de cuan bueno sea usted y de la buena reputación que tengan sus productos o servicios, no puede dejar de mejorar. Cuando lo hace, realmente no se ha detenido, sino que retrocede, porque su competencia mejora constantemente"(Harrington, 1997).

Seguidamente expresa un grupo de razones por las que hay que mejorar los procesos:

- Todos los días surgen nuevos métodos, programas y equipos.

- El ambiente de la empresa cambia, por lo que los procesos eficientes se hacen obsoletos de la noche a la mañana.

- Las expectativas del consumidor y del cliente cambian casi todos los días; lo que fue sobresaliente ayer escasamente satisface las necesidades de hoy y resulta inadecuado mañana.

- Las personas vinculadas al proceso incrementan sus capacidades, e implica un aumento de oportunidades para la depuración del proceso.

- Los procesos descuidados o no atendidos se degradan con el transcurso del tiempo.

- Independientemente de lo bueno que sea hoy el proceso, siempre habrá un camino mejor. Todo lo que necesita hacer es descubrirlo.

Varios autores en la divulgación de sus obras, emiten conceptos relacionados a la mejora continua. En los inicios estaban estrechamente relacionados a la calidad (Ishikawa, 1988) (Juran, 1990) y ya se reconocía la necesidad del compromiso de todo el personal (Demmig, 1989). Aunque es considerado un cambio ventajoso tiene sus inicios en una creación organizada (Imai, 1995), no es un producto del azar(Formento, 2002). Por su parte, Harrington (1997) introduce el concepto ligado a la obtención de procesos más efectivos, eficientes y adaptables, unos años después la ISO 9000:2000 lo ratifica al planteárselo como una actividad recurrente para aumentar 
la capacidad de cumplir requisitos. Otros (Flores Ripoll, 2010) (Aguilar Morales, 2010) lo vinculan como elemento fundamental para la implantación de los sistemas gestión normalizados, se lo plantean como el aprendizaje continuo de la organización, donde nada puede considerarse como algo terminado de forma definitiva.

La mejora continua constituye un cambio organizado para obtener niveles superiores de desempeño. Donde necesariamente no tiene que iniciarse por incumplimiento o daño del sistema instalado, el deseo de alcanzar estándares superiores puede tributar a un enfoque proactivo que sirva como motor impulsor de la rueda de mejora.

También Harrington (1997) divulga diez requisitos que hacen exitoso el proceso de mejora, deja implícito, a través del requisito cuatro, su carácter proactivo:

1. La aceptación del consumidor como la cuestión más importante del proceso.

2. Compromiso de largo plazo de la dirección para hacer el proceso de mejoramiento parte del sistema de dirección.

3. Creer que hay espacio para mejorar.

4. Creer que prevenir los problemas es mejor que reaccionar ante ellos.

5. Enfoque de la dirección, liderazgo y participación.

6. El estándar de desempeño de cero errores.

7. La participación de todos los empleados, tanto de los grupos como de los individuos.

8. El enfoque del mejoramiento sobre el proceso, no sobre la gente.

9. Creer que los suministradores trabajarán con usted y comprenden sus necesidades.

10. Reconocimiento de los éxitos.

Se pueden sintetizar entonces, algunos beneficios de la mejora de procesos:

1. Se disminuyen recursos (materiales, personas, dinero, mano de obra), y aumenta la eficiencia.

2. Se disminuyen tiempos, y aumenta la productividad.

3. Se disminuyen errores, se ayuda a prevenirlos.

4. Se ofrece una visión sistemática de las actividades de la organización.

La norma ISO 9004:2009 (ISO, 2009) plantea que las actividades de mejora pueden variar desde pequeñas mejoras continuas en el lugar de trabajo hasta las más significativas en toda la organización, se aplica de manera coherente con el enfoque basado en proceso y la organización debe asegurarse que se establezca como parte de la cultura de la organización.

En síntesis, los proyectos de mejora se tratan en la bibliografía con tres aristas, una dada por la mejora paulatina o continua, otra por saltos, o simplemente por una combinación entre ellas.

\subsubsection{Mejora Continua}


La mejora continua se logra a través de todas las acciones diarias (por pequeñas que éstas sean) que permiten que los procesos y la empresa sean más competitivos en la satisfacción del cliente. La velocidad del cambio dependerá del número de acciones de mejora que se lleven a cabo día a día y de la efectividad con que éstas se realicen, por lo que es importante que sea una idea enraizada en la conducta de todos los miembros de la organización, convirtiéndose en una filosofía de trabajo, de vida.

Sin embargo, mejorar no es solo cuestión de buenos deseos e intenciones. Las personas tienen que estar convencidas del beneficio que en lo individual obtendrán al adoptarla. La organización tiene la responsabilidad de proporcionarles motivación para tal efecto, además de procedimientos estandarizados y técnicas de análisis apropiadas para que puedan materializar y orientar correctamente sus deseos de mejorar.

El proceso de mejora continua pretende cambiar la forma de pensar de las personas acerca de los errores. Para ello existen dos maneras de reforzar la aplicación de los cambios deseados: reprender a todos los que no logren hacer bien su trabajo o premiar los individuos y grupos cuando alcancen una meta y realicen un importante aporte al proceso de mejora. Un sistema de así, requiere descansar en la habilidad de los miembros de una empresa para reconocer las oportunidades de mejora.

La mejora continua, como lo enfatiza el enfoque japonés, debe verse como una filosofía de vida que tiene por objetivo perfeccionar a las personas y, por consecuencia, el medio en que se desarrollan, que cada vez es más exigente.

En la tabla 2 se muestran ventajas y desventajas que este enfoque supone. Obsérvese como a pesar de sus reconocidas ventajas en cuanto a los plazos para la obtención de resultados, la acumulación de cambios obliga a saltos superiores de mejora en el futuro.

Tabla 2: Ventajas y desventajas de la mejora continua.

\begin{tabular}{|c|c|}
\hline & \\
\hline $\begin{array}{l}\text { - Se centra el esfuerzo en elementos } \\
\text { organizativos y de procedimientos puntuales. } \\
\text { - Proporciona recursos para preparar los } \\
\text { procesos a los nuevos adelantos } \\
\text { tecnológicos. } \\
\text { - Posibilita la eliminación de procesos } \\
\text { repetitivos. } \\
\text { - Logra el establecimiento de niveles de } \\
\text { desempeño superiores necesario para las } \\
\text { actuales organizaciones. } \\
\text { - Se obtienen mejoras y resultados visibles en } \\
\text { un corto plazo. } \\
\text { Si se reduce el porcentaje de defectuosos, se } \\
\text { obtienen una reducción en los costos como } \\
\text { resultado de un consumo menor de materias } \\
\text { primas. }\end{array}$ & $\begin{array}{l}\text { - Sus aplicaciones han sido centradas al campo de } \\
\text { la mejora de la calidad. Con bajo nivel a la } \\
\text { integración de sistemas de gestión y alineación con } \\
\text { las estrategias. } \\
\text { - Para el logro exitoso se requiere de la atención y el } \\
\text { conocimiento de toda la empresa. } \\
\text { - La acumulación de cambios, fruto de la } \\
\text { implementación de mejoras continuas en el tiempo, } \\
\text { obligan a saltos superiores en el diseño de los } \\
\text { procesos. } \\
\text { - Cuando el mejoramiento se realiza con un carácter } \\
\text { funcional o por áreas se limita la interdependencia } \\
\text { que existe entre el resto de los miembros de la } \\
\text { empresa. } \\
\text { - Obliga en muchas ocasiones a ejecutar inversiones } \\
\text { constantemente. }\end{array}$ \\
\hline
\end{tabular}




\subsubsection{Mejora Radical o reingeniería}

Durante la década de los 90s se popularizó el término reingeniería de negocios (Babé, 2002.), debido principalmente a que algunas empresas vieron en este concepto la manera de lograr un salto rápido de los procesos que deterioran su competitividad, para los que la mejora continua resulta una forma muy lenta de avanzar.

Independientemente de lo que muchas personas piensan, este enfoque de mejora no es realmente nuevo. Bajo el enfoque japonés adopta el nombre de innovación o cambio radical (Ishikawa, 1988). De manera que lo que hoy se conoce como reingeniería es una conceptualización de la innovación japonesa bajo el enfoque occidental de gestión.

La mejora radical o reingeniería no se pude considerar como un proceso de bajo riesgo (Hammer, \& Champy, 1993). Añade también Ignacio Babé (2002) que significa trabajo duro, que lleva tiempo (no son arreglos rápidos).

De acuerdo con Champy (1995) existen cuatro aspectos que se deben considerar en un estudio de reingeniería si se desea tener éxito: el cuestionamiento constante sobre el propósito y la razón de ser de todo lo que se hace en la organización, el cambio cultural que se necesita en los niveles organizacionales y la definición del tipo de personal que se requiere para ello, la creación de nuevos procedimientos, normas y estándares, y la puesta en práctica de los nuevos procedimientos.

La realidad es que cuando se implementa un cambio radical y seguido a este no se aplican un conjunto de mejoras continuas, el rendimiento no permanece constante, incluso, disminuye, por tanto no deben absolutizarse por separados, ni un programa de reingeniería, ni un programa de mejora continua, sino debe ser el resultado de una aplicación continua, o sea, un programa de reingeniería siempre debe estar precedido y subseguido por una serie de mejoras continuas, y así sucesivamente(Cantú, 2001).

La mejora es un proceso ajustado a las necesidades de cada organización y del análisis profundo y consciente dependerá cual camino escoger.

\subsection{Consideraciones acerca de los procedimientos de mejora de procesos}

En la bibliografía existe un número importante de herramientas encaminadas a la gestión y mejora de los procesos, unas más específicas y otras de mayor espectro; clasificadas en metodologías, modelos y procedimientos. Un punto en común es tener como base el ciclo de mejora PHVA, fundamentalmente por su carácter cíclico y por las distintas ventajas brindadas (Ishikawa, 1988)(Comas Rodríguez et al., 2014):

- Asegura un programa en el que se ha convenido para la terminación del proyecto. 
- Asegura el análisis, la verificación y la eliminación de los modos de fallos más probables.

- Facilita la puesta en práctica de controles para supervisar y administrar el nuevo proceso mejorado.

- Crea las condiciones para la capacitación permanente y la actualización de la documentación que se requiere en cada ciclo de mejora.

- Evita la reaparición de las causas que provocan los problemas, mediante la estandarización de los procesos mejorados.

Esta diversidad de herramientas para la gestión y mejora viene dada fundamentalmente, porque cada empresa o institución en general tiene características distintas, aunque sus producciones (salidas) tengan similitud. El entorno, el personal, el mercado, el presupuesto, constituyen elementos que pueden influir para hacer que cada programa de mejora deba realizarse como un traje a la medida. A pesar de ello, resulta enriquecedor examinar los procedimientos que con este fin aparecen en la literatura especializada. En estudios realizados (Medina León, et al., 2012) se aprecia una evolución sustancial en los procedimientos de mejora de procesos desde finales del siglo veinte hasta la fecha. En los comienzos eran más generales y terminaban por omitir medidas concretas para la implementación de acciones de mejora. Con el de cursar del tiempo y con nuevos conceptos en la gestión empresarial comenzaron a ser más específicos y orientadores, muchos de ellos constituyen trajes a la medida para sectores, aunque no dejan de sentar las bases para su posible adaptación en otros.

Sin embargo, existen puntos en común en muchos de ellos:

- Coinciden en el carácter cíclico de la mejora.

- En la identificación de los procesos empresariales.

- En la importancia de nombrar responsables de procesos y de las acciones de mejora.

- En el establecimiento de indicadores para el control.

Por otro lado también difieren en elementos que la propia evolución exige en la actualidad:

- Se orienta la mejora a partir de los requisitos del sistema de gestión de calidad, sin explicitar otros sistemas que operan.

- Determinar las oportunidades resulta un paso decisivo en la mejora. Es aquí donde muchos autores difieren en la manera de realizarla; unos parten del banco de problema o de situaciones emergentes, otros, del análisis de cumplimiento al sistema de indicadores establecidos para el logro de la estrategia empresarial. Se observa que la inclinación por una u otra vía dependen; para la primera, si se diseña un procedimiento para ser aplicado como mecanismo de intervención, generalmente por agentes externos. Para la segunda, si se diseña para ser aplicado internamente, donde se requiere un nivel superior de trabajo alcanzado por el personal de la institución 
- Otro aspecto escasamente formalizado es la evaluación de las posibles acciones de mejora. La práctica señala que no todas se pueden aplicar al unísono, ya sea por razones de tiempo, monetarias, disponibilidad de personal 0 , incluso, porque perjudique la eficiencia o la eficacia de otros sistemas de la organización.

- La atención a la resistencia al cambio debiera trabajarse con una mayor intensidad, si se entiende como un elemento esencial en la implantación.

- Los mecanismos de retroalimentación escasamente explicitan el carácter cíclico del modelo de mejora.

Por tanto, resulta imprescindible el diseño de modelos y procedimientos de mejora de la gestión de los procesos, ajustados a las nuevas necesidades de todas las partes interesadas y a los requerimientos propios de la entidad donde se implementen.

\subsection{Los sistemas de gestión empresariales, su normalización}

La calidad constituye un elemento importante en el posicionamiento de las empresas en el mercado y llega a ser esencial para la supervivencia. Esto forma parte del conocimiento de los directivos y personal en general; se le presta especial interés a lo largo de la historia, pero no siempre fue así y los enfoques cambian.

A principios de la década del cincuenta el aseguramiento de la calidad nació como una evolución natural del control de calidad, que resultaba limitado y poco eficaz para prevenir la aparición de defectos. Por esta razón, se hizo necesario crear sistemas de calidad que incorporasen la estructura, las responsabilidades, actividades, recursos y procedimientos para llevar a cabo la gestión de la calidad. Con esta finalidad y para estandarizar los sistemas de calidad se comenzó a publicar en 1987 las normas ISO 9000 (Martínez Llebrez, 2015).

Cronológicamente y sobre la base de que los modelos de excelencia constituyen un paso superior en el peldaño empresarial, fuertemente relacionado con la mejora continua y la Gestión por Procesos, surge en mil novecientos noventa y seis la ISO 14001 ligada a la protección medioambiental. Esto constituye la evidencia más contundente de la necesidad que tenía el medio de responder a otros factores además de la calidad, ya se había comenzado a pensar en otras partes que también demandaban respuestas de las empresas.

Sin embargo, otros sistemas de gestión (SG) como el de Seguridad e Higiene del Trabajo despertaban poco interés, debido en parte a la ausencia de un estándar ISO en esa área. Este organismo había anunciado en la primavera de mil novecientos noventa y cinco (1995) la creación de un grupo de trabajo cuyo objetivo fuera consensuar la posibilidad de desarrollo de dicho estándar, pero comunicó en enero de mil novecientos noventa y siete (1997) a través de su Technical Management Board que no iniciaría actividades en ese momento en el campo de los 
estándares de Seguridad e Higiene del Trabajo; y hasta hoy todavía no las ha iniciado (Abad Puente, 2011).

Con vistas a cubrir el vacío e incentivados por la necesidad, muchos organismos de certificación nacionales tomaron la iniciativa y surge de este modo las OHSAS (Occupational Health and Safety Assessment Series). En la actualidad es el estándar dominante a nivel internacional para certificación del sistema de Seguridad e Higiene del Trabajo (Granerud \& Rocha, 2011)(Abad Puente, Lafuente, \& Vilajosana Crusells, 2013), aunque también existe las Directrices de la Organización Internacional del Trabajo (OIT) (Abad Puente, 2011), enunciadas entre los años mil novecientos noventa y nueve (1999) y dos mil uno (2001).

Por la importancia otorgada, en el año dos mil cuatro (2004) se publica la revisión actualizada de la ISO 14001, en el dos mil siete (2007), la revisión de la OHSAS 18001 y en el dos mil ocho (2008) las ISO 9001. La evolución de los sistemas no se detiene, en el dos mil nueve se lanza una revisión de la ISO 9004 y en el dos mil once (2011) se publica una nueva norma la ISO 50001 dedicada al consumo energético, elemento importante para el desempeño empresarial actual; un año más tarde se lanza la revisión de la ISO 9001, en el 2015 aparece la aprobación y publicación de la nueva versión de la ISO 9001(Martínez Llebrez, 2015).

Es importante señalar que las normas mencionadas no son las únicas, pero si las más empleadas en la integración de sistemas de gestión(Abad Puente, Rodríguez Mondelo, \& Sánchez-Toledo, 2011); aunque también se integra la gestión de la innovación, la responsabilidad social corporativa y en Cuba el sistema de gestión de capital humano.

Un análisis a estas normas evidencia la similitud entre ellas; vale agregar que tienen como base la Gestión por Procesos y la mejora continua.

\subsection{Sistemas Integrados de Gestión. Tendencias Internacionales}

Desde hace algunos años se realizan estudios a los sistemas de gestión (calidad, medio ambiente y seguridad y salud del trabajo), cómo se implementan y evolucionan de forma satisfactoria en las organizaciones, con la intención de proponer modelos de gestión que ayuden a procurar la satisfacción de sus diferentes partes interesadas, aunque de forma separada (Abad Puente et al., 2011).

Los sistemas mencionados, a pesar de ser los más referidos en la literatura, no son los únicos. También existen SG fundamentados en otros estándares internacionales que son desarrollados por la Organización Internacional de Normalización (ISO), por ejemplo: ISO/IEC 27001 Seguridad de la información e ISO/IEC 20000 Gestión de Servicios TI. Estadísticas anuales develan un crecimiento saludable de las certificaciones en todos los ámbitos(Gasiorowski, 2013). 
Así pues, en la gestión empresarial coexisten dos tendencias complementarias dentro las organizaciones: la implantación de sistemas de gestión a partir de los correspondientes estándares nacionales e internacionales, que facilitan de forma separada el desempeño en cada una de las funciones técnicas asociadas a los diferentes stakeholders (partes interesadas) y el diseño de esquemas de excelencia empresarial que garanticen la satisfacción de forma integrada de todos los grupos de interés implicados(Karapetrovic, 2002).

La existencia de las normas Internacionales da lugar a que numerosas organizaciones implementen y certifiquen los SG de forma independiente, en paralelo; esto provoca semejanzas en el contenido y estructuras. Pero cada día son más conscientes de las desventajas que trae consigo operar sistemas en paralelo (Santos, Mendes, \& Barbosa, 2011), por tanto abogan por tener un único sistema de gestión (Simon, Karapetrovic, \& Casadesus, 2012).

De lo anterior y en coincidencia con Abad Puente (2009) es de cuestionarse el mantener sistemas de gestión separados y generar con ello las consecuentes duplicidades y sub-optimizaciones de recursos. Los estándares se fundamentan en los principios de gestión (mejora continua, gestión por procesos, alta implicación de la dirección) y comparten esquemas y requisitos similares (formación de los trabajadores, auditorías internas, definición de objetivos), por lo que la integración constituye una vía a formularse para obtener un único sistema de gestión o un Sistema Integrado de Gestión (SIG). También (Fraguela Formoso, Carral Couse, Iglesias Rodríguez, Castro Ponte, \& Rodriguez Guereiro, 2011) plantea la necesidad de la integración como elemento fundamental para satisfacer las necesidades, expectativas y requisitos de las partes interesadas.

Según la norma (UNE 66177, 2005) se define el SIG como "la parte de la gestión general de la organización que determina y aplica la política integrada de gestión, y que surge de la integración de las gestiones de la calidad, el medio ambiente y la seguridad y salud del trabajo".

Autores como (Peña Escobio, Rigol Cardona, \& Moreno Pino, 2009) coinciden al definir un sistema de gestión integrada como "la parte del sistema de gestión empresarial para establecer las políticas y objetivos con respecto a varios aspectos (en este caso la calidad, el medio ambiente y la seguridad y salud en el trabajo), así como la planificación, implementación y control de las acciones para lograrlas".

De García (De García, 2009) añade la responsabilidad social corporativa y menciona que: la gestión integrada "no es más que una nueva forma de enfocar las actividades de una organización para gestionar integralmente la variable calidad, ambiente, seguridad y salud ocupacional y responsabilidad social corporativa; tiene como propósito el logro de una política integrada de gestión. Es una forma de responder a las nuevas exigencias técnicas en los mercados nacionales e internacionales". 
Añade (Bernardo, Casadeus, Karapetrovic, \& Heras, 2009) que un SIG es "un conjunto de procesos interconectados que comparten un grupo de humanos, de información, materiales, infraestructura y recursos financieros con el fin de lograr una combinación de objetivos relacionados con la satisfacción de una gran variedad de interesados".

Por su parte, la autoras Conzález (2003) e Issac Godínez (2007) definen un SGI como el conjunto de la estructura organizativa, la planificación de las actividades, las responsabilidades, las prácticas, los procedimientos, los procesos, y los recursos necesarios para desarrollar, implantar, llevar a efecto, revisar y mantener al día la política de la empresa.

Se puede inferir, entonces, que un sistema integrado de gestión es un proceso que transita diferentes fases que cumplen con los requisitos establecidos para un único sistema de gestión, basándose en la política, procedimientos, implementación, seguimiento y control, auditorías y mejoras. Esto permite a la organización demostrar su compromiso hacia todas las partes interesadas.

Cuando se desea un SIG se requiere que la organización establezca los procesos dentro de su alcance, de forma tal que den respuesta a los propósitos de los sistemas que son objeto de integración. Con un enfoque preventivo que considere los aspectos de impacto y riesgos asociados a sus actividades y resultados, debido a que la integración de sistemas no significa una suma o adición de SG.

El objetivo es sistematizar todos los procesos de la organización y en mayor medida los procesos claves y relevantes que intervienen; con el propósito de lograr un nivel de integración en la gestión para así aumentar la eficiencia y la eficacia. Además, proporciona a las organizaciones un mejor resultado empresarial al integrar los sistemas, los procesos que los soportan y las actividades que componen los procesos (Cuendias de Armas et al., 2013)

Hoy día no existe una norma internacional ISO que determine los requisitos de un sistema de gestión integrada de calidad, medio ambiente y seguridad y salud en el trabajo; sin embargo con todas las normas y requisitos establecidos para cada SG, se considera cada vez más lógico la creación de una norma ISO para la integración de los sistemas (Abad Puente, 2011).

Tal necesidad ha condicionado a que varios organismos de certificación de carácter nacional realicen propuestas metodológicas de normas, que es preciso conocer en el momento de realizar un proyecto de integración. Dentro de ellas: una norma española (AENOR), UNE 66177:2005 que proporciona directrices para desarrollar, implantar y evaluar el proceso de integración de los SG de la calidad, medio ambiente y gestión de la seguridad y salud de los trabajadores.

Otra propuesta es la australiana denominada "Integración de los SG. Guía para Empresas, Gobierno y Organizaciones Comunitarias (AS/NZS 4581) ". También el British Standard Institution 
(BSI) del Reino Unido lanza la guía sobre la implementación de los SG integrados HB 10190 IMS: The framework y la especificación disponible al público PAS 99:2006: Especificación de requisitos comunes del SG como marco para la integración, la cual fue adoptada por la Oficina Nacional de Normalización (ONN) como documento normativo en 2008 para la certificación en Cuba, (Cuendias de Armas et al., 2013), donde al igual que las otras normas dice el qué hacer, pero no el cómo llegar a la implantación.

De estudios realizados en varias regiones de Europa, entre ellas Italia (Salomone, 2008), España (Bernardo et al., 2009) y (Karapetrović et al., 2010), en las pequeñas y mediana empresas del Reino Unido (Douglas \& Glen, 2000); y en otras regiones del mundo China (Zeng, Shi, \& Lou, 2007), Australia (Zutshi \& Sohal, 2005), se puede resumir que muchas integran particularmente los SG de calidad, luego medio ambiente y por último seguridad y salud en el trabajo, así como también medio ambiente y después calidad. En menor cuantía se suman otros SG.

En general, los sistemas de gestión certificables que más se valoran actualmente para los procesos de integración son: sistema de gestión de la calidad, sistema de gestión ambiental y sistema de gestión de seguridad y salud en el trabajo. En los últimos años, se añade el sistema energético.

Se debe tener presente, que el principal motor de la integración son las propias organizaciones y no agentes externos: clientes, autoridades, entre otros, como ocurre generalmente con los sistemas de gestión individuales(Castañeira, 2010).

\section{Conclusiones y Recomendaciones}

- En la actualidad cualquier intento por formalizar la gestión y mejora de procesos debe contener supuestos teóricos alrededor de la gestión por procesos y la integración de sistemas. Abriendo así, una nueva línea para futuros trabajos científicos.

- Existe coincidencia en los procedimientos de conducción de la gestión y mejora de procesos en cuanto a la presentación cíclica y la preparación por fases y etapas.

- Resulta imprescindible aceptar las buenas prácticas de gestión tratadas con un enfoque de procesos, que resaltan en la selección de los procesos, la determinación y evaluación de las oportunidades de mejora, los indicadores y su relación con las estrategias empresariales, tratados implícitamente o no en los procedimientos tomados como antecedentes.

\section{Bibliografía}

Abab Puente, J., Vilajosana Crusells, J., \& Dalmau, I. (2014). Taxonomic proposal for integration levels of management systems based on empirical evidence and derived corporate benefits. 
Journal of Cleaner Production, XXX. Recuperado a partir de http://dx.doi.org/10.1016/j.jclepro.2014.04.084

Abad Puente, J., Lafuente, E., \& Vilajosana Crusells, J. (2013). An assessment of the OHSAS 18001 certification process: Objective drivers and consequences on safety performance and labour productivity. Journal of Cleaner Production, 47-56.

Abad Puente, Jesús. (2009, diciembre). "Aspectos clave de la integración de sistemas de gestión”, 10-12.

Abad Puente, Jesús. (2011, junio). Implicaciones de la integración de los sistemas de Gestión de Calidad, Medio Ambiente y Seguridad y Salud Laboral basados en estándares internacionales (Tesis Doctoral.). Universidad Politecnica de Catalunya.

Abad Puente, Jesús, Rodriguez Mondelo, P., \& Sánchez-Toledo, A. (2011). Management systems integration Spanish survey reveals key benefits. ISO Focus +, 2(1), 42-45.

Aguilar Morales, J. E. (2010). La mejora continua. Asociación Oaxaqueña de Psicología. Recuperado a partir de www.conductitlan.net

Aiteco Consultores. (2002). Gestión por procesos. Recuperado a partir de www.aiteco.com

Alonso Torres, C. (2014). Orientaciones para implementar una gestión basada en procesos. Ingeniería Industrial, XXXV(2), 161-172.

Álvarez Suarez, A. (2011). Gestión por procesos. Ediciones de la Universidad de Oviedo.

Amozarrain, M. (1999). La Gestión por Procesos. España: Mondragón Corporación Cooperativa.

APQC. (2012, julio). Process classification framework. APQC. Recuperado a partir de www.apqc.org

Azcanio García, A., QuintanaTápanes, L. Q., Medina León, A., \& Nogueira Rivera, D. (2006). «Tendencias de la Gestión Empresarial relacionadas con procesos». Gestiopolis. Recuperado a partir de HTTTUhttp://www.gestiopolis.com/canales7/ger/gestion-empresarialrelacionada- con-procesos.htmUTTH

Babé, I. (2002, abril). RE-INGENIERÍA: ¿ARREGLO RÁPIDO O SOLUCIÓN A LARGO PLAZO? Juran Institute España, S.A.

Balcázar, H., \& Lagunas, P. (2013). Importancia del análisis de los procesos de una organización para el cumplimiento de los requisitos de ISO 9001:2000 y para la mejora de su desempeño. Recuperado a partir de http://www.bvsde.paho.org/bvsacd/cd29/analisis.pdf 
Bernardo, M., Casadeus, M., Karapetrovic, S., \& Heras, I. (2009). "How integrated are environmental, quality and other standardized management systems. An empirical study", (17), 742-750.

Bratić, D. (2011). Six Sigma: A Key Driver for Process Improvement. IBIMA, 15. doi:10.5171/2011. 823656

Bravo Carrasco, J. (2009). Gestión de Procesos. Desde la mejora hasta el rediseño. (Vols. 1-I, Vol. I). Santiago. Chile: Evolución. Recuperado a partir de www.evolucion.cl

Cantú, H. D. (2001). Desarrollo de una cultura de Calidad. México: Editorial Mc Graw- Hill.

Castañeira, R. (2010). Diseño del Sistema Integrado de Gestión para la Empresa de Diseño e Ingeniería Las Tunas V. I. Lenin: 85.

Champy, J. (1995). Reengineering Management: The Mandate for the New Leadership. Harper Business.

Comas Rodríguez, R., Nogueira Rivera, D., Medina León, A., Romaro Bartutis, F., \& Lumpuy Rodriguez, M. (2014, julio). La evaluación del alineamiento estratégico en las organizaciones. IX Congreso Internacional de Gestión Empresarial y Administración Pública y el V Taller Internacional de Escuelas y Facultades de Capacitación de Directivos. GESEMAP, 2014.

Conzález, A. (2003). "Material de la Maestría de Calidad Total".

Cordoví Cuza. (2013). La mejora continua a partir de la implantación del sistemalntegrado de Gestiónen la ECOING 5. En Memorias del XIII Encuentro TECNOGEST 2013.Gestión del Conocimiento y Empresas de Alto Desempeño. Tecnología, Innovación y Desarrollo Sostenible. La Habana. Cuba: TECNOGEST 2013.

Cuendias de Armas, J., suárez Palou, H., Brito Álvarez, Z., Pérez Acosta, M., Pérez Ménde, L., Pevida Fernández, T., ... Ayala Ávila, I. (2013). "Manejo integrado de Gestión". Cubaenergía.

Davenport, T. . (1993). Process Innovation. Reengineering work through information technology. Harvard business School Press, Borton.

De García. (2009). "Gestión Integrada: una moda o una exigencia técnica para nuestras empresas".

Demmig, E. (1989). Calidad, productividad y competitividad. España.

Díaz Gorino. (2002). La Gestión por Procesos. Recuperado a partir de www.jcedes.com 
Douglas, A., \& Glen, D. (2000). Integrated management systems in small and medium enterprises. Total Quality Management, 11, 686-690.

Flores Ripoll, M. V. (2010). La Mejora Continua en empresas y organizaciones. Cronicas de la mejora continua. Recuperado 12 de septiembre de 2015, a partir de http://www.eoi.es/blogs/mariavictoriaflores/definicion-de-mejora-continua/feed/

Formento H. (2002). Modelo para la aplicación de un proceso de mejora sistemática. Recuperado a partir de www.gestiondelacalidad.com.ar

Fraguela Formoso, J., Carral Couse, L., Iglesias Rodríguez, G., Castro Ponte, A., \& Rodriguez Guereiro, M. J. (2011). La integración de los Sistemas de gestión. Necesidad de una nueva cultura empresarial. Open Journal Systems, 78(167), 44-49.

Gasiorowski, E. (2013). Latest ISO Survey confirms boost in management systems (2013-10-24) ISO. Recuperado 19 de febrero de 2014, a partir de file:///D:/Latest\%20ISO\%20Survey\%20confirms\%20boost\%20in\%20management\%20syste ms\%20(2013-10-24)\%20-\%20ISO.htm

González Méndez, L. (2002). El Enfoque de Procesos. Recuperado a partir de www.uh.cu/centros/ceec/Enfoque de procesos.

Granerud, L., \& Rocha, R. . (2011). Organisational learning and continuous improvement of health and safety in certified manufacturers. Saf. Sci, 49(7). Recuperado a partir de www.refhub.elseiver.com/s0959-6526(14)00449-1/sref21

Grieco, P. L. (1997). World Class. Excelencia empresarial. Ediciones Deusto, S.A., Barcelona.

Hammer, M., \& Champy, J. (1993). Reengineering the Corporation: A Manifiesto for Business Revolution. Nueva York: Harper Business.

Harrington, H. James. (1997). Administración Total del Mejoramiento Continuo: la Nueva Generación. Colombia: Editorial Mc Graw-Hill.

Hernández, A. (2003). Gestión por proceso. Revista Ingeniería Industrial, 35(1).

Hernández Nariño, A, Nogueira Rivera, D., Medina León, A., \& Marqués León, M. (2013). Insertion of business process management in health care organizations: methodological and practical conception. Revista de Administração, 48(4), 739-756.

Hernández Nariño, Arialys, Medina León, A., Nogueira Rivera, D., \& Marqués León, M. (2009). Mejora y perfeccionamiento de los procesos hospitalarios. Propuesta de un algoritmo para su aplicación. Avanzada Científica, 12(No 1). 
IFA. (2010). Handbook of International Quality Control, auditing ,review, other assurance, and related services pronouncements. New York, USA: International Federation of Accountants.

Imai, M. K. (1995). Kaizen: la clave de la ventaja competitiva japonesa. México.

Ishikawa, K. (1988). ¿Qué es el control de la calidad? La modalidad japonesa. Cuba.

ISO. (2009, noviembre). Norma Internacional ISO 9004:2009. ISO. Recuperado a partir de www.iso.org

ISO. (2015). Risk-based thinking in ISO 9001:2015. International Organization for Standardization. Recuperado a partir de www.iso.org/tc176/sc02/public

ISO 9001: 2008. (s. f.). Norma Internacional ISO 9001: 2008. Secretaria Central de ISO. Recuperado a partir de www.iso.org

Issac Godínez, C. L. (2007). "Guía práctica para la identificación y evaluación de aspectos ambientales en la organización. Identificación de aspectos ambientales".

Junginger, C. (2000). «La Gestión por Procesos en organizaciones sanitarias»; Recuperado a partir de www.ujasalud.com

Juran, \& Blanton. (2001). Manual de calidad (5 ed.). Mc Graw Hill.

Juran, J. M. (1990). Juran y el liderazgo para la calidad: Un manual para directivos. España.

Karapetrovic, S. (2002). "Strategies for the integration of management systems and standards". The TQM Magazine, Vol.14(1), 61-67.

Karapetrovic, S. (2003). "Musing on integrated management systems". Measuring Bussines Excellence, 7(1), 4-13.

Karapetrović, S., Casadesus, M., \& Heras, I. (2010). Empirical analysis of integration within the standards-based INTEGRATED MANAGEMENT SYSTEMS. International Journal for Quality Research, 4(2). Recuperado a partir de www.ijqr.net/journal/v4-n1/3.pdf

Maldonado, J. A. (2011). Gestión de Procesos. Recuperado a partir de www.eumed.net/librosgratis/2011e/1084/indice.htm

Martínez Llebrez, V. R. (2015, marzo). El nuevo proyecto ISO/DIS 9001 Sistemas de Gestión de Calidad. Requisitos. Su aprobación como norma internacional en el 2015. Presentacion en Power Point, TECNOGEST.

Medina Léon, A., Nogueira Rivera, D., Comas Rodríguez, R., Hernández Nariño, A., Ricardo Cabrera, H., \& Medina Nogueira, D. (2014). La Ficha de Proceso, soporte del enfoque de 
procesos y del control de gestión. IX Congreso Internacional de Gestión Empresarial y Administración Pública y el V Taller Internacional de Escuelas y Facultades de Capacitación de Directivos. GESEMAP, 2014.

Medina León, A., Nogueira Rivera, D., Hernández Nariño, A., \& Viteri, J. (2010). Relevancia de la gestión por procesos en la Planificación Estratégica y la Mejora Continua. EIDOS, 2, 18.

Medina León, A, Nogueira Rivera, D, \& Hernández Nariño, A. (2012, diciembre). Consideraciones y criterios para la selección de procesos para la mejora: Procesos Diana. Ingenieria Industrial, XXXIII(3), 272-281.

Mora Martínez, J. . (2002). «Gestión Clínica por Procesos: mapa de procesos de enfermería en centros de salud». Revista de Administración Sanitaria, VI(21). Recuperado a partir de www.dinarte.es/ras/ras21/pdf/09\%20adm\%20y\%20gest\%204.pdf.

Morcillo Ródenas, C. (2000). «Gestión por Procesos en Andalucía: ¿Qué aportan?» Samfyc. Recuperado a partir de www.samfyc.es/nueva/revista/PDF/v3n3/01.pdf

Moreira, M. (2013). La gestión por procesos en las instituciones de información. ACIMED, 14(5). Recuperado a partir de http://scielo.sld.cu/scielo.php?script=sci_arttext\&lng=pt\&nrm=iso\&tlng=pt\&pid=S1024943520 06000500011

Nariño Hernández, A., Nogueira Rivera, D., \& Medina León, A. (2014). La caracterización y clasificación de sistemas, un paso necesario en la gestión y mejora de procesos. Particularidades en organizaciones hospitalarias. DYNA, 81(184), 191-198.

Nogueira Rivera, D. (2002). «Modelo conceptual y herramientas de apoyo a la toma de decisiones para potenciar el Control de Gestión en las empresas cubanas»; (Tesis presentada en opción al grado científico de Doctor en Ciencias Técnicas.). Universidad de Matanzas.

Paneque Sosa, P. (2002). Gestión por Procesos en el sistema sanitario público de Andalucía. Curso de formación Médica continuada. Huercal- Overa. Recuperado a partir de www.medynet.com/usuarios/jraguilar/gestion_procesos.pdf

Peña Escobio, D., Rigol Cardona, B., \& Moreno Pino, M. (2009). Proyeccion de Sistemas Integrados de Gestión. Contribuciones a la Economía. Recuperado a partir de www.eumed.net/ce/2009a/ecp.htm

Pepper Bergholz, S. (2011, mayo). Definición de Gestión por Procesos. Medwave, XI(No 5), 3.

Ponjuán Dante. (2005). Principios y métodos para el mejoramiento organizacional. Ciudad de La Habana. Cuba: Editorial Félix Varela. 
Rey Peteiro, D. (2012). Todo sobre la Gestión por Procesos (Parte I). Sinapsys Business Solutions, FUNDIBEQ. Recuperado 12 de septiembre de 2015, a partir de www.w3.org/TR/xhtml1/DTD/xhtml1-strict.dtd

Ricardo Cabrera, H. (2009, octubre). Procedimiento para la mejora continua de los procesos de la Empresa de Productos Lácteos Escambray. Universidad de Cienfuegos «Carlos Rafael Rodríguez».

Ricardo Cabrera, H. (2010). Aplicación de un procedimiento de mejora a procesos ordenados secuencialmente a partir de métodos multicriterios. Recuperado a partir de www.eumed.net/libros/2010a/650

Salomone, R. (2008). Integrated management systems: experiences in Italian organizations. Journal of Cleaner Production, (16), 1786-1806.

Santos, G., Mendes, F., \& Barbosa, J. (2011). Certification and integration of management systems: the experience of Portuguese small and medium enterprises. Journal of Cleaner Production, 19, 17-18.

SESCAM. (2002). La Gestión por Procesos. Recuperado a partir de www.chospab.es/calidad/UCalidad/Documentos/Gestiondeprocesos.pdf.

Simon, A., Karapetrovic, S., \& Casadesus, M. (2012). Evolution of integrated management systems in Spanish firms. Journal of Cleaner Production, 23(1), 8-19.

Solé Cabanes, A. (2012). Gestión por procesos. Recuperado a partir de www.asolengin.bubok.es

Trischler, W. E. (2000). Mejora del valor añadido en los procesos.

UNE 66177. (2005). "Sistemas de gestión. Guía para la integración de los sistemas de gestión”.

Zaratiegui, J. R. (1999). "La Gestión por Procesos: su papel e importancia en la empresa" (Vol. VI). España: Economía Industrial.

Zeng, S. ., Shi, J., \& Lou, G. X. (2007). A synergetic model for implementing an integrated management system: an empirical study in China. Journal of Cleaner Production, (15), 17601767.

Zutshi, A., \& Sohal, A. S. (2005). Integrated management system. The experiences of three Australian organisations. Journal of Manufacturing Technology Management, 16, 211-232. 